\title{
System Identification of the Linac RF System Using A Wavelet Method and Its Applications in the SNS LLRF Control System*
}

\author{
Yi-Ming Wang, Sung-Il Kwon, Amy Regan, and Tony Rohlev, LANL, Los Alamos, NM 87545 USA
}

\begin{abstract}
For a pulsed LINAC such as the SNS, an adaptive feed-forward algorithm plays an important role in reducing the repetitive disturbance caused by the pulsed operation conditions. In most modern feed-forward control algorithms, accurate real time system identification is required to make the algorithm more effective. In this paper, an efficient wavelet method is applied to the system identification in which the Haar function is used as the base wavelet. The advantage of this method is that the Fourier transform of the Haar function in the time domain is a sinc function in the frequency domain. Thus we can directly obtain the system transfer function in the frequency domain from the coefficients of the time domain system response.
\end{abstract}

\section{INTRODUCTION}

The Spallation Neutron Source (SNS) Linac to be built at Oak Ridge National Laboratory (ORNL) consists of a combination of low energy normal conducting (NC) accelerating structures as well as higher energy superconducting RF (SRF) structures. The purpose of RF system modeling is to investigate the various cavity configurations in order to provide the correct requirements for the RF control system hardware. We use modeling as a way to specify RF components; verify system design and performance objectives; optimize control parameters; and to provide further insight into the RF control system operation.

In control applications such as iterative learning feedforward control, the available a priori model information is often not sufficiently accurate to allow a prior fixed design that satisfies all the performance specifications. In these circumstances, the designer may reduce the specified performance level, expand additional efforts to reduce the amount of model uncertainty or design a mechanism to reduce the amount of the model uncertainty by using the input-output data.

The system identification techniques can be divided into two categories [1]: parametric and nonparametric. Parametric techniques assume that the functional form of the system model is known but that the model parameters are unknown. Nonparametric techniques are necessary when the functional form is unknown. In this case, a general family of functional approximators is selected based on the known properties of the approximation family and the characteristics of the application. Therefore, the designer must specify an appropriate family

${ }^{\circ}$ Work supported by the Office of Basic Energy Science, Office of Science of the US Department of Energy, and by Oak Ridge National Laboratory. of approximators, specify the structure of the approximators, and estimate the parameter values to optimize the function approximation. This paper will focus on the nonparametric system identification using wavelet.

\section{WAVELET OVERVIEW}

A wavelet is a small wave which has its energy concentrated in time to give a tool for the analysis of transient, nonstationary, or time-varying phenomena [2]. It still has oscillating wave-like characteristics but also has the ability to allow simultaneous time and frequency analysis with a flexible mathematical foundation. In Fourier series, a signal can be represented by a series expansion of sinusoids. In the same way, a signal can be represented by a series expansion of wavelets. As in Fourier series, a discrete-time series expansion is introduced.

Consider a vector space $V$. If any $f(t) \in V$ can be expressed by

$$
f(t)=\sum_{k} a_{k} \varphi_{k}(t),
$$

the set of functions $\varphi_{k}(t)$ is called an expansion set for the space $V$ and if the representation is unique, the set is a basis. Alternatively, consider a basis set and define a space $V$ as the set of all functions that can be expressed by $f(t)=\sum_{k} a_{k} \varphi_{k}(t)$. This is called the span of the basis set.

Define a set of scaling functions which are integer translates of the basic scaling function $\phi(t) \in L^{2}$, namely,

$$
\phi_{k}(t)=\phi(t-k), k \in Z \text {. }
$$

The subspace of $L^{2}$ spanned by these translated functions is defined as

$$
V_{0}=\overline{\operatorname{span}\left\{\phi_{k}(t)\right\}} \text {, }
$$

for all $k \in Z$. This means that $f(t)=\sum_{k} a_{k} \phi_{k}(t)$ for any $f(t) \in V_{0}$. A two-dimensional family of functions is generated from the basic scaling function by scaling and translation by

$$
\phi_{j, k}(t)=2^{j / 2} \phi\left(2^{j} t-k\right)
$$

whose span over $\mathrm{k}$ is

$$
V_{j}=\overline{\operatorname{span}\left\{\phi_{j, k}(t)\right\}}
$$

For the wavelet expansion, a two-parameter system is constructed such that the expansion for all $k \in Z$. This means that $f(t)=\sum_{k} a_{k} \phi\left(2^{j} t-k\right)$ for any $f(t) \in V_{j}$. Extending this idea, the following relation is obtained.

$$
\cdots \subset V_{-2} \subset V_{-1} \subset V_{0} \subset V_{1} \subset V_{2} \subset \cdots \subset L^{2} \text {. }
$$


This relation is the requirement of the multiresolution analysis (MRA) which is frequently used for a signal approximation. The nesting of the spans of $\phi\left(2^{j} t-k\right)$ denoted by $V_{j}$ is achieved by requiring that $\phi(t) \in V_{1}$, which means that if $\phi(t)$ is in $V_{0}$, it is also in $V_{1}$, the space spanned by $\phi(2 t)$ which implies that $\phi(t)$ is the solution of

$$
\phi(t)=\sum_{n} h(n) \sqrt{2} \phi(2 t-n), \quad n \in Z
$$

where $h(n)$ is the scaling function coefficients to be solved.

The important features of a signal can better be described not by using $\phi_{j, k}(t)$ and increasing $j$ to increase the size of the subspace spanned by the scaling functions, but by defining a slightly different set of functions $\psi_{j, k}(t)$ that span the differences between the spaces spanned by the various scales of the scaling function. These functions are the wavelets. Usually, the scaling functions and the wavelets are orthogonal. Orthogonal basis functions allow simple calculation of expansion coefficients and have Parseval's theorem that allows a partitioning of the signal energy in the wavelet transform domain. The orthogonal complement of $V_{j}$ and $V_{j+1}$ is defined as $W_{j}$.

$$
V_{j+1}=V_{j} \oplus W_{j} .
$$

This means that all members of $V_{j}$ are orthogonal to all members of $W_{j}$. In addition, it is required that

$$
\left\langle\phi_{j, k}(t), \psi_{j, l}(t)\right\rangle=\int \phi_{j, k}(t), \psi_{j, l}(t) d t=0
$$

for all appropriate $j, k, l \in Z$.

From (6) and (8), the space $L^{2}$ is represented by

$$
L^{2}=V_{j 0} \oplus W_{j 0} \oplus W_{j 0+1} \oplus W_{j 0+2} \oplus \cdots
$$

The prototype wavelet functions is given by the form

$$
\psi_{j, k}(t)=2^{j / 2} \psi\left(2^{j} t-k\right)
$$

where $2^{j}$ is the scaling of $t, 2^{-j} k$ is the translation of $t$, and $2^{j / 2}$ maintains the $L^{2}$ norm of the wavelet at different scales. $\psi(t)$ is the solution of

$$
\psi(t)=\sum_{n} h_{1}(n) \sqrt{2} \phi(2 t-n), \quad n \in Z
$$

for some set of coefficients $h_{1}(n)$.

With the scaling function $\phi_{j, k}(t)$ and the wavelet $\psi_{j, k}(t)$, a signal $g(t) \in L^{2}$ can be written by

$$
g(t)=\sum_{k} c_{j 0}(k) \phi_{j, k}(t)+\sum_{k} \sum_{j=j 0}^{\infty} d_{j}(k) \psi_{j, k}(t)
$$

The first term of the right hand side is the coarsest scale whose space is spanned by $\phi_{j, k}(t)$ and the second term of the right hand side is the high resolution details of the signal $g(t)$ which is spanned by the wavelets $\psi_{j, k}(t)$. The coefficients of the expansion (10) are called the discrete wavelet transform (DWT) of the signal $g(t)$ which can be calculated by inner products

$$
\begin{aligned}
& c_{j 0}(k)=\left\langle g(t), \phi_{j, k}(t)\right\rangle \\
& d_{j}(k)=\left\langle g(t), \psi_{j, k}(t)\right\rangle
\end{aligned}
$$

For the general wavelet expansion, Parseval's theorem is

$$
\int|g(t)|^{2} d t=\sum_{l=-\infty}^{\infty}|c(l)|^{2}+\sum_{j=0}^{\infty} \sum_{k=-\infty}^{\infty}\left|d_{j}(k)\right|^{2}
$$

with the energy in the expansion domain partitioned in time by $k$ and in scale by $j$.

\section{HAAR WAVELET SYSTEM}

If a scaling function is chosen to have compact support over $0 \leq t \leq 1$, then the solution $\phi(t)$ of (7) is [3],

$$
\phi(t)=\left\{\begin{array}{cc}
1, & 0 \leq t \leq 1 \\
0 & \text { otherwise }
\end{array}\right.
$$

with only two nonzero scaling function coefficients $h(0)=h(1)=\frac{1}{\sqrt{2}}$, and the wavelet, the solution of is

$$
\psi(t)=\left\{\begin{array}{cc}
1, & 0<t<0.5 \\
-1, & 0.5<t<1 \\
0 & \text { otherwise }
\end{array}\right.
$$

with only two nonzero wavelet coefficients $h_{1}(0)=\frac{1}{\sqrt{2}}$, $h_{2}(1)=-\frac{1}{\sqrt{2}}$. See figure $1 . V_{0}$ is the space spanned by $\phi(t-k)$, the next higher resolution space $V_{1}$ is spanned by $\phi(2 t-k)$. As higher values of scale $j$ are considered, the space $V_{j}$ is spanned by $\phi\left(2^{j} t-k\right)$ and becomes a better approximation of arbitrary functions or signals by finer and finer piecewise constant functions.

Using only scaling function does not allow the detailed decomposition of a signal. For the higher resolution decomposition of a signal, the wavelet is necessary. The basic wavelet of a Haar wavelet system is an oscillating function which has an average of zero and produces finer and finer detail as it scaled and translated. For example, consider the scale $j=3$. Since

$$
\begin{aligned}
& V_{3}=V_{2} \oplus W_{2}, \\
& V_{2}=V_{1} \oplus W_{1}, \\
& V_{1}=V_{0} \oplus W_{0},
\end{aligned}
$$

a smooth signal can be approximated from the basis elements in

$$
V_{3}=V_{0} \oplus W_{0} \oplus W_{1} \oplus W_{2} .
$$


Since Haar functions form an orthogonal basis in each subspace, they can produce an optimal least squared error approximation to the smooth signal.
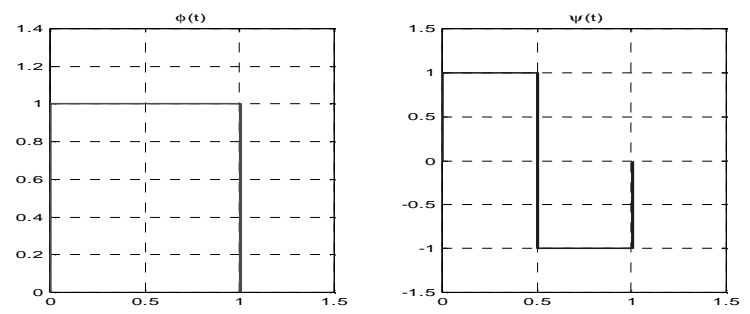

Figure 1 Scaling function and wavelet function of Haar Wavelet

\section{HAAR WAVELET SYSTEM APPLICATION}

The open loop low level RF system for a SRF cavity can be represented by two input two output (TITO) system in baseband of I/Q coordinates. The off diagonal terms of the input matrix are very small compared to the diagonal terms [4]. The coupling between I channel and Q channel is mainly due to the detuning caused by beam loading, the Lorentz Force Detuning, and microphonics. In frequency domain, the transfer matrix of the low level RF system can be represented by

$$
\left[\begin{array}{l}
Y_{I}(z) \\
Y_{Q}(z)
\end{array}\right]=\left[\begin{array}{ll}
H_{I I}(z) & H_{I Q}(z) \\
H_{Q I}(z) & H_{Q Q}(z)
\end{array}\right]\left[\begin{array}{c}
U_{I}(z) \\
U_{Q}(z)
\end{array}\right],
$$

where $H_{n m}(z), m, n=I, Q$, is the Z-transform of the impulse response $h_{n m}(t), \quad m, n=I, Q$ from the input $u_{m}(t), \quad m=I, Q$, to the output $y_{n}(t), n=I, Q$. In order to obtain the system characteristics, system identification is necessary. Parametric system identification obtains the coefficients of the transfer matrices $H_{n m}(z), m, n=I, Q$, explicitly and nonparametric system identification obtains the functional form of system input-output relation which is usually given by the impulse response $h_{n m}(t)$, $m, n=I, Q$. It is not difficult to obtain the approximate transfer matrix $H_{n m}(z)$ from the impulse response data $h_{n m}(t)$.

Let $h(t)$ be a discrete time impulse response of an arbitrary input channel to an arbitrary output channel. Then, it can be represented by Haar approximations in various resolutions. Figure 2 shows the impulse response $h_{I I}(t)$ and its Haar function approximations in various resolutions, where 1024 point sequence $h_{I I}(t)$ is obtained from Matlab/Simulink model of low level RF system with sampling time $50 \mathrm{nsec}$. The numbers of scaling function coefficients are $(32,64,128,256,512)$ for $j=0,1,2,3,4$. Note that Haar functions are orthogonal basis and can produce an optimal least squared error approximation to the smooth signal. For example, consider $j=0$. Then, $h_{I I}(t)$ is approximated as

$$
h_{I I}(t) \approx \sum_{k=0}^{31} c_{00}(k) \phi(t-k)
$$

where the scaling function coefficients are obtained from (13) with $\phi_{0, k}(t)=\phi(t-k)$ and shown in figure 3. Ztransform of both side of (20) yields the approximation of the transfer function $H_{I I}(z)$.

$$
H_{I I}(z) \approx \sum_{k=0}^{31} c_{00}(k) Z(\phi(t-k))=\sum_{k=0}^{31} c_{00}(k) z^{-k} Z(\phi(t))
$$

where $Z(\phi(t))$ is Z-transform of $\phi(t)$.
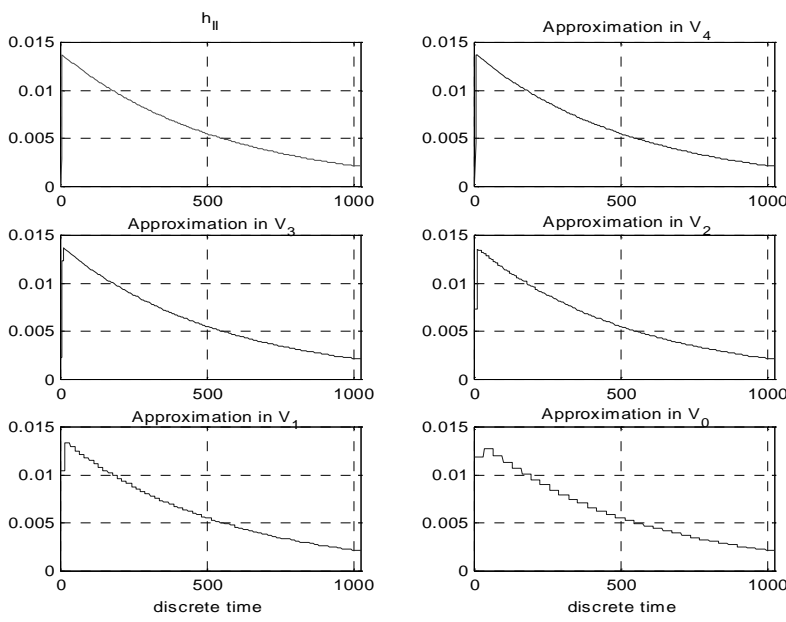

Figure 2 Haar function approximations

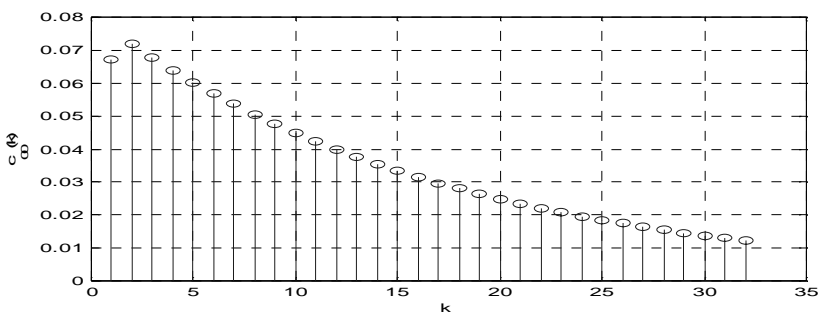

Figure 3 Approximation Coefficients in $V_{0}$

\section{CONCLUSION}

In this paper, Haar wavelet system is applied to approximate the impulse response of a system. Due to the characteristics of Haar functions, the approximation of the transfer function of the system is easily obtained where the coefficients of the transfer function are the discrete wavelet transform of the impulse response.

\section{REFERENCES}

[1] N. Sureshbabu and J. Farrell, "Wavelet Based System Identification for Nonlinear Control Applications," Proceedings of IEEE Control and Decision Conference, pp. 236-241, 1995.

[2] S. Mallat, A Wavelet Tour of Signal Processing, Academic Press, 1998.

[3] M. Doroslovacki and H. Fan, "Wavelet-Based Linear System Modeling and Adaptive Filtering," IEEE Trans. Signal Processing, Vol. 44, No. 5, pp. 1156-1167, 1996.

[4] S. Kwon, et al, "Uncertain System Modeling of SNS RF Control System", PAC’01, Chicago, June 2001. 